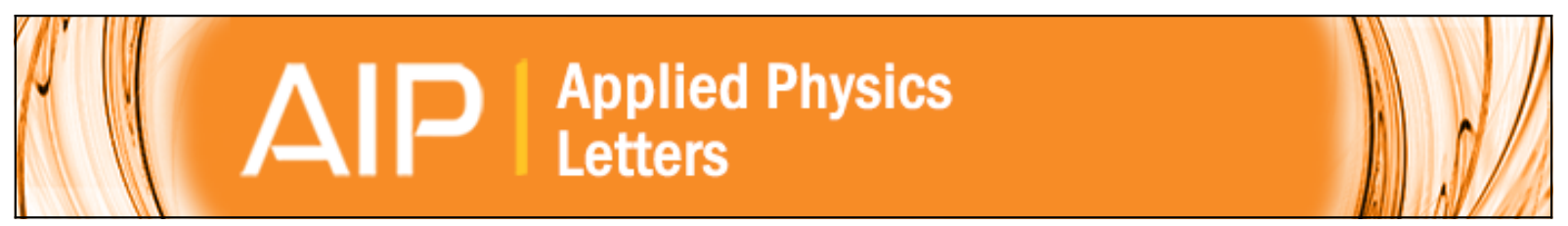

\title{
Hyperelastic tension of graphene
}

E. I. Saavedra Flores, R. M. Ajaj, S. Adhikari, I. Dayyani, M. I. Friswell, and Rafael Castro-Triguero

Citation: Applied Physics Letters 106, 061901 (2015); doi: 10.1063/1.4908119

View online: http://dx.doi.org/10.1063/1.4908119

View Table of Contents: http://scitation.aip.org/content/aip/journal/apl/106/6?ver=pdfcov

Published by the AIP Publishing

\section{Articles you may be interested in}

Thermal vibration of a rectangular single-layered graphene sheet with quantum effects

J. Appl. Phys. 115, 233515 (2014); 10.1063/1.4885015

Effects of loading mode and orientation on deformation mechanism of graphene nano-ribbons

Appl. Phys. Lett. 103, 191906 (2013); 10.1063/1.4829480

Identification of micro parameters for discrete element simulation of agglomerates

AIP Conf. Proc. 1542, 871 (2013); 10.1063/1.4812070

Graphene bubbles with controllable curvature

Appl. Phys. Lett. 99, 093103 (2011); 10.1063/1.3631632

van der Waals adhesion of graphene membranes

J. Appl. Phys. 107, 044301 (2010); 10.1063/1.3270425

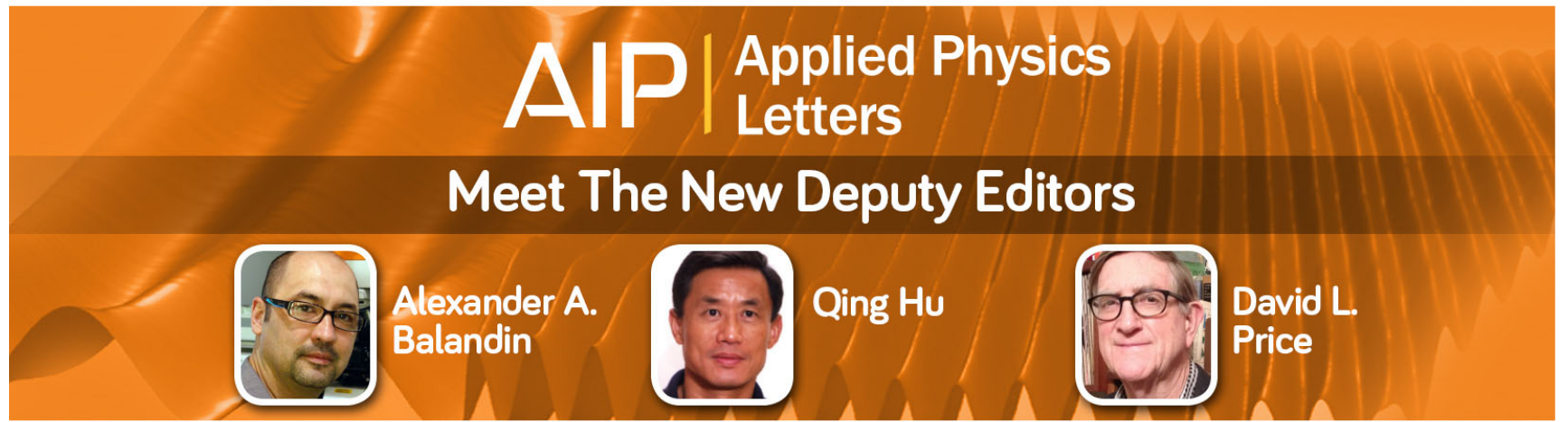




\title{
Hyperelastic tension of graphene
}

\author{
E. I. Saavedra Flores, ${ }^{1, a)}$ R. M. Ajaj, ${ }^{2}$ S. Adhikari, ${ }^{3}$ I. Dayyani, ${ }^{3}$ M. I. Friswell, ${ }^{3}$ \\ and Rafael Castro-Triguero ${ }^{4}$ \\ ${ }^{1}$ Departamento de Ingeniería en Obras Civiles, Universidad de Santiago de Chile, Av. Ecuador 3659, \\ Santiago, Chile \\ ${ }^{2}$ Aeronautics and Astronautics, University of Southampton, Southampton SO171BJ, United Kingdom \\ ${ }^{3}$ College of Engineering, Swansea University, Singleton Park, Swansea SA2 8PP, United Kingdom \\ ${ }^{4}$ Department of Mechanics, University of Cordoba, Campus de Rabanales, Cordoba CP 14071, Spain
}

(Received 23 September 2014; accepted 3 February 2015; published online 9 February 2015)

\begin{abstract}
In this paper, we investigate the hyperelastic tensile behaviour of single layer graphene sheets (SLGSs). A one-term incompressible Ogden-type hyperelastic model is chosen to describe the mechanical response of $\mathrm{C}-\mathrm{C}$ bonds. By establishing equality between the Ogden strain-energy and the variation of the Tersoff-Brenner interatomic potential, three different geometries of SLGSs are studied under tensile loading. We compute the Young's modulus, the finite-deformation Poisson's ratio, ultimate strains, total reactions, and the variation of the potential energy per carbon atom for large strains. Numerical simulations are compared with results obtained by molecular mechanics and molecular dynamics simulations, finite elements, continuum mechanics theory, and experiments. Our predictions are validated, revealing the potential predictive capabilities of the present hyperelastic framework for the analysis of graphene in the context of infinitesimal and large deformations. The good agreement found between our calculations and the published data suggests that graphene may be described as a hyperelastic material. (C) 2015 AIP Publishing LLC.
\end{abstract}

[http://dx.doi.org/10.1063/1.4908119]

Over the past decade, the investigation of single layer graphene sheets (SLGSs) has brought substantial progress, particularly in the understanding of their mechanical behaviour by means of computational predictive simulations. In the context of numerical methods, the finite element (FE) based lattice approach, or atomistic FE method, is a promising technique to analyse the mechanical response of complex nanostructures. ${ }^{1}$

The atomistic FE method establishes an equivalence between structural and molecular mechanics (MM) at the $\mathrm{C}-\mathrm{C}$ bond level, representing a versatile procedure to model atomic bonds by means of beam-type elements in conventional FE analyses.

Due to its mathematical simplicity and potential adaptability to describe the response of several materials within the regime of large strains, an Ogden hyperelastic isotropic material model ${ }^{2}$ is chosen for the non-linear mechanical description of C-C bonds. We note that a hyperelastic behavior of SLGSs has been observed recently. ${ }^{3}$ Experimental evidence of the non-linear effects in SLGSs has also been reported. ${ }^{4}$

A generic finite hyperelastic model is characterised by the existence of a strain energy density function $\Psi$ which is determined uniquely by a tensor describing the deformation of the material. If this deformation is defined by the principal stretches $\lambda_{i}$ (with $i=1,2,3$ ) or eigenvalues of the right (and left) stretch tensor, ${ }^{2}$ the constitutive equation for the principal Kirchhoff stresses can be written as ${ }^{2}$

$$
\tau_{i}=-p+\lambda_{i} \frac{\partial \Psi}{\partial \lambda_{i}}
$$

\footnotetext{
a) Author to whom correspondence should be addressed. Electronic mail: erick.saavedra@usach.cl
}

Here, perfect incompressibility has been assumed. The scalar $p$ in Eq. (1) is identified as a hydrostatic pressure and may be determined only from equilibrium equations and the boundary conditions.

In this work, a one-term incompressible version of the Ogden strain-energy density function is adopted. This can be expressed as

$$
\Psi=\frac{2 \chi}{\alpha^{2}}\left\{\left(\lambda_{1}\right)^{\alpha}+\left(\lambda_{2}\right)^{\alpha}+\frac{1}{\left(\lambda_{1} \lambda_{2}\right)^{\alpha}}-3\right\} .
$$

We note that in the above equation, the volumetric constraint $\lambda_{1} \lambda_{2} \lambda_{3}=1$ has been adopted. The parameters $\chi$ and $\alpha$ are material constants and are obtained by fitting Eq. (2) according to the variation of the Tersoff-Brenner interatomic potential. ${ }^{5,6}$ By adopting an equivalent beam element for each C-C bond, with circular cross-section of diameter $0.147 \mathrm{~nm}$ and equilibrium bond length of $0.142 \mathrm{~nm},{ }^{7}$ the above fitting procedure results in the following material constants:

$$
\chi=1166.18 \mathrm{GPa} \text { and } \alpha=-10.0,
$$

for those $\mathrm{C}-\mathrm{C}$ bonds under longitudinal shortening, and

$$
\chi=2332.36 \mathrm{GPa} \text { and } \alpha=-14.0,
$$

for those bonds under longitudinal stretching.

We use the commercial software ABAQUS (Ref. 8) and we analyse three arbitrary geometries with initial dimensions of $4.67 \mathrm{~nm} \times 4.11 \mathrm{~nm}, 3.44 \mathrm{~nm} \times 1.99 \mathrm{~nm}$, and $1.48 \mathrm{~nm}$ $\times 1.56 \mathrm{~nm}$, in the $X$ and $Y$ (in-plane) directions, respectively. For the first SLGS, the corresponding FE mesh consists of 780 nodes and 1131 beam elements. The second mesh contains 290 nodes and 411 elements, and the third mesh, 104 
nodes and 142 elements. We select the two-noded hybrid beam element, type $\mathrm{B} 31 \mathrm{H}$, which is compatible with the adoption of a hyperelastic Ogden model. ${ }^{8}$ Transverse shear strains are considered in the beam element formulation. Non-linear kinematics (and the non-linear response of the material under a large strains regime) is considered in the modelling. Although Timoshenko B31H beams can be subjected to large non-linear axial strains, their transverse shear behavior is assumed to be linear elastic with a fixed modulus. Vertical displacements (along the $Y$ direction) are set to zero on the nodes located at $Y=0$ (bottom edge). Horizontal displacements (along the $X$ direction) are also set to zero at lower and upper left nodes. Prescribed displacements in the vertical direction are imposed on the upper edge, and increase from zero up to the onset of the breakage of the C-C bonds. Refer to Figure 1 for details.

In order to validate the present model, we compute the Young's modulus for the three cases analysed. The numerical predictions presented here are obtained from the linearised elastic constants of our model. After straightforward mathematical procedures, ${ }^{9}$ the value of the constant $\chi$ in Eq. (2) can be related to the linear elastic shear modulus $G$, through the condition $\chi=G .^{10,11}$ In the limit of incompressibility, the shear modulus becomes $E / 3$ in isotropic solids. Thus, we obtain the relationship $E=3 \chi$, which is used to perform our computational simulations under small displacement theory and an infinitesimal strain regime.

In Table I, our numerical predictions are compared with published data, taking into account several theoretical techniques, such as FE simulations, ${ }^{12-14}$ molecular dynamics (MD) ${ }^{15}$ and density functional theory (DFT) ${ }^{16,17}$ In order to be consistent with these data, our calculations considered an equivalent thickness of $0.34 \mathrm{~nm}$. It is also interesting to compare our values with the experimental measures of $1.0 \mathrm{TPa}$ (Refs. 4 and 18) and 0.5 TPa (Ref. 20) (AFM). An experimental value of 1.0 TPa was also reported for pyrolytic graphite. ${ }^{19}$ In the context of carbon nanotubes, Yu et al. ${ }^{21}$ measured values between $0.32 \mathrm{TPa}$ and $1.47 \mathrm{TPa}$. Ding et $a l^{22}$ determined values between 0.62 and $1.2 \mathrm{TPa}$ (SEM).

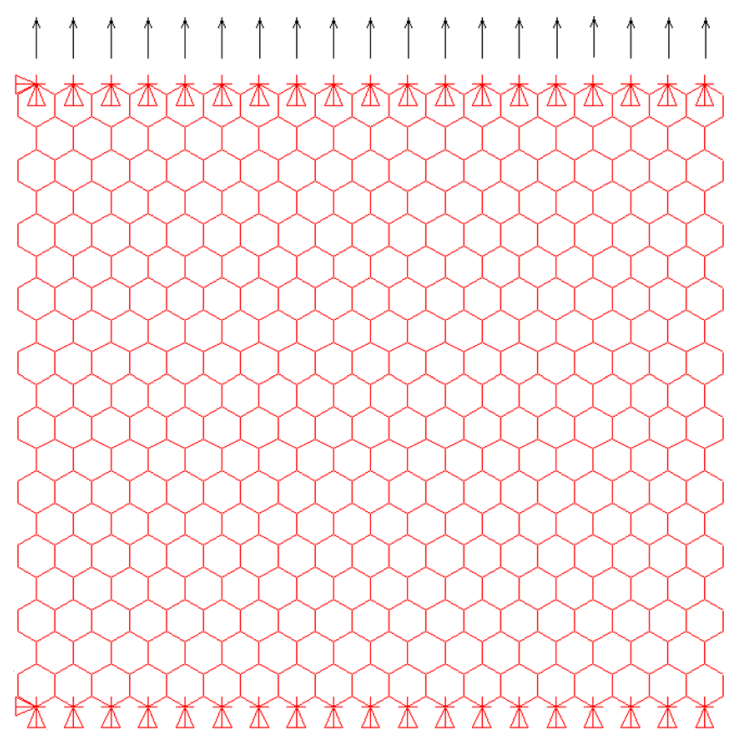

FIG. 1. Typical FE mesh of a SLGS with initial dimensions of $4.67 \mathrm{~nm} \times$ $4.11 \mathrm{~nm}$, along the horizontal $(X)$ and vertical $(Y)$ directions, respectively.
TABLE I. Comparison between published values for the Young's modulus and our numerical predictions.

\begin{tabular}{lcc}
\hline \hline Value (TPa) & Comments & References \\
\hline 0.722 & FE simulations & 12 \\
0.74 & FE simulations & 13 \\
1.0 & FE simulations & 14 \\
0.72 & MD simulations & 15 \\
0.82 & DFT & 16 \\
1.05 & DFT & 17 \\
1.0 & Experiments & $4,18,19$ \\
0.5 & Experiments & 20 \\
$0.32-1.47$ & Experiments & 21 \\
$0.62-1.2$ & Experiments & 22 \\
0.822 & $4.67 \mathrm{~nm} \times 4.11 \mathrm{~nm}$ SLGS & Present model \\
0.81 & $3.44 \mathrm{~nm} \times 1.99 \mathrm{~nm}$ SLGS & Present model \\
0.80 & $1.48 \mathrm{~nm} \times 1.56 \mathrm{~nm}$ SLGS & Present model \\
\hline \hline
\end{tabular}

We must note that, in spite of having a relatively good agreement with those values less than $0.85 \mathrm{TPa}$, our predicted results are smaller than those values next to $1 \mathrm{TPa}$.

From Table I, we also observe a size-dependence that our model is able to predict. Size-dependent elastic properties of graphene have been previously reported. ${ }^{23,24}$ For instance, Jing et al. ${ }^{24}$ reported that the Young's modulus of graphene increases from $0.7 \mathrm{TPa}$ up to a saturated value of 1.1 $\mathrm{TPa}$ as the size of the graphene samples increases. Importantly, the same increasing trend can be observed in our numerical predictions up to a saturated value of 0.836 TPa (simulation not shown here) as the length of the sample increases.

We note that the small differences found in the mechanical properties of SLGSs are due to the fact that graphene sheets are non-homogeneous materials and therefore their properties are dependent on the choice of their dimensions. The ability of our model to capture these differences, and therefore the discrete nature of graphene, represents one of the main advantages of the present approach when compared to continuum mechanics-based models, which normally fail to reproduce these effects.

We also validate our hyperelastic model by comparing the total Ogden strain-energy obtained from straining each SLGS and then dividing by the total number of nodes with the variation of the interatomic potential energy per carbon atom obtained from several sources. ${ }^{25-28}$ Figure 2 shows this comparison. In this graph, $\varepsilon_{y}$ refers to the overall strain of the graphene sheets along the $Y$-direction and is defined as $\varepsilon_{y}=L_{y} / L_{o y}-1$, with $L_{y}$ and $L_{o y}$ the current and initial lengths in the $Y$-direction, respectively. The corresponding curves show a good agreement up to 0.16 strain between our numerical predictions and the results reported in Ref. 25 (atom-based cell model), and in Refs. 26 and 27 (MD), and those results reported in Ref. 28 (MM), obtained for GNRs with widths $(W)$ of 1.2 and $2.5 \mathrm{~nm}$.

In order to introduce a failure criterion, several cut-off distances have been adopted in the literature, with no significant changes in the critical bond-breaking force for values ranging from $0.17 \mathrm{~nm}$ to $0.19 \mathrm{~nm} .{ }^{29}$ In this study, we adopt a value of $0.19 \mathrm{~nm}$, which is interpreted physically as the onset of the breakage of the $\mathrm{C}-\mathrm{C}$ bonds under stretching. Here, we 


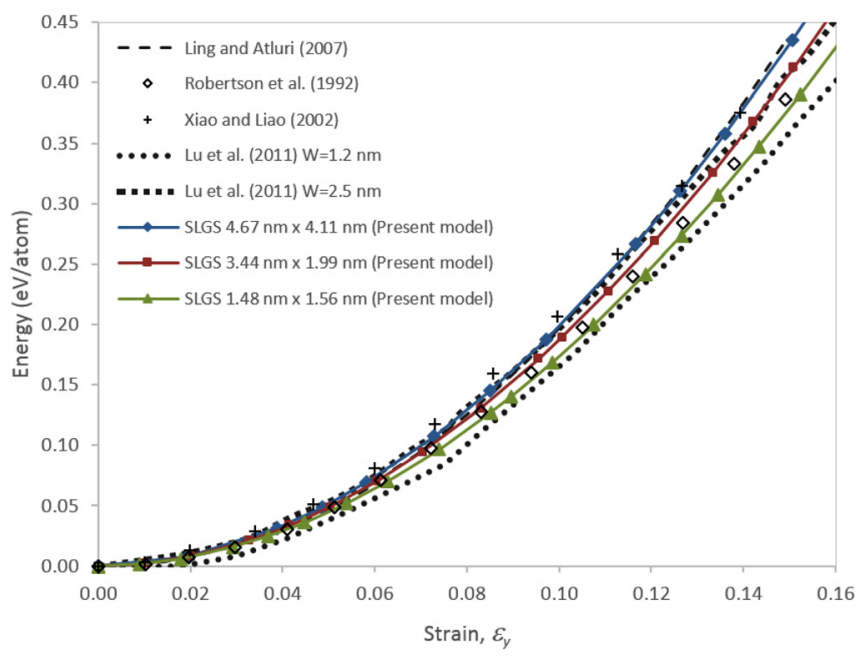

FIG. 2. Potential energy per carbon atom as a function of the overall strain $\varepsilon_{y}$ for different geometries under uniaxial tension, ${ }^{25-28}$ along with the total Ogden strain-energy obtained from our numerical simulations.

assume that the failure of at least one $\mathrm{C}-\mathrm{C}$ bond implies the failure of the whole SLGS.

Table II shows our numerical predictions for the ultimate strains of SLGSs and some values found in the literature. The comparison again shows a good agreement with those values published in Refs. 12 and 30 (FE), in Ref. 28 (MM), and Ref. 4 (experiments).

The total vertical reaction at the bottom line of nodes of the three SLGSs is plotted as a function of the maximum interatomic extension of $\mathrm{C}-\mathrm{C}$ bonds in Figure 3. Here, we can note that the three mechanical responses show an almost linear variation, with a slight decrease in the slope of the curves at about 0.03 strain. For larger strains, over 0.15 , a slight increase of the slope can be observed, which is attributed to the beginning of the geometric alignment of the $\mathrm{C}-\mathrm{C}$ bonding elements along the loading direction. This effect is possible to observe since large strains/displacements have been taken into account in our computational simulations. Here, the maximum overall strain in each of the graphene sheets is 0.29 along the $Y$-direction. A second reason for this effect is the use of the Tersoff-Brenner formulation as the interatomic potential, which provides a stiffer mechanical response compared to other standard potentials, such as the Morse formulation. ${ }^{31}$ In Figure 3, the maximum interatomic extension of the C-C bonds increases up to the failure of the SLGS, at 0.19 strain.

We also explore in this paper that the variation of the finite-deformation Poisson's ratio, $\nu$, in each of the SLGSs.

TABLE II. Summary of values published for the ultimate strains of SLGSs and our numerical predictions.

\begin{tabular}{lcc}
\hline \hline Value & Comments & References \\
\hline 0.28 & FE simulations & 12 \\
0.25 & FE simulations & 30 \\
0.25 & Experiments & 4 \\
0.29 & MM simulations & 28 \\
0.291 & $4.67 \mathrm{~nm} \times 4.11 \mathrm{~nm}$ SLGS & Present model \\
0.294 & $3.44 \mathrm{~nm} \times 1.99 \mathrm{~nm}$ SLGS & Present model \\
0.295 & $1.48 \mathrm{~nm} \times 1.56 \mathrm{~nm}$ SLGS & Present model \\
\hline \hline
\end{tabular}

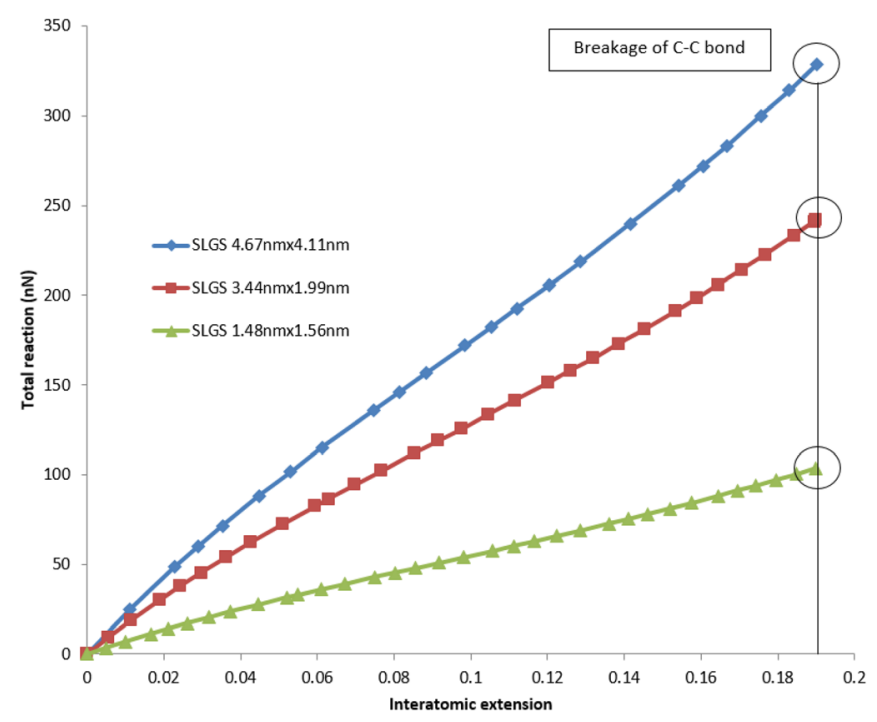

FIG. 3. Total vertical reaction at the bottom line of nodes as a function of the maximum interatomic extension of the C-C bonds for the three SLGSs.

This parameter is defined as the ratio between the in-plane lateral contraction (along the $X$-direction) and the in-plane extension parallel to the loading direction (along the $Y$-axis), that is, $\nu=-\varepsilon_{x} / \varepsilon_{y} .{ }^{17}$ Therefore, the term "finitedeformation Poisson's ratio" refers here to a measure of the overall change in the dimensions of the graphene sheet, but not to a property of the material itself. We note that in the modelling of the $\mathrm{C}-\mathrm{C}$ bonds, we have assumed a value of 0.5 for the Poisson's ratio of the equivalent material as a consequence of adopting the perfect incompressibility constraint $\lambda_{1} \lambda_{2} \lambda_{3}=1$ in Eq. (2).

Figure 4 shows the variation of the finite-deformation Poisson's ratio in each of the three SLGSs as the strain $\varepsilon_{y}$ increases up to total failure. At small strains, the three curves approach a Poisson's ratio of 0.41 , approximately. This value agrees with the Poisson's ratio of 0.412 (Ref. 32) (finite deformation continuum theory), the value of 0.416 (Ref. 33) (continuum mechanics approach), the value of 0.413 (Ref. 34) (MD), and 0.398 (Ref. 35) (nonlinear continuum mechanics).

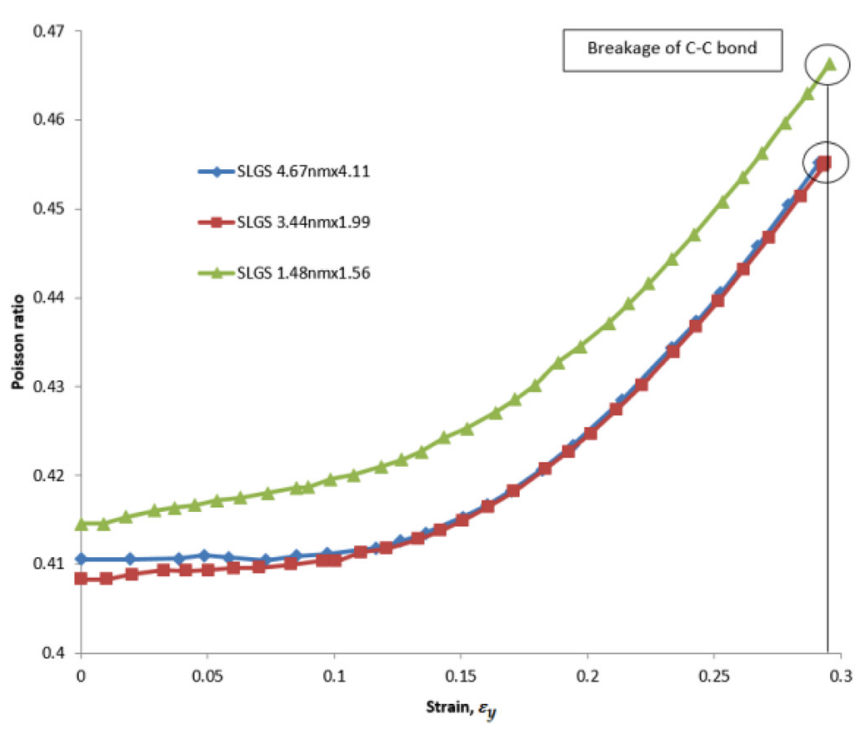

FIG. 4. Variation of the finite-deformation Poisson's ratio in each of the three SLGSs as the overall strain $\varepsilon_{y}$ increases. 
TABLE III. CPU times involved in the computational simulations of four SLGSs by means of the present approach.

\begin{tabular}{lcc}
\hline \hline SLGS dimensions $(\mathrm{nm} \times \mathrm{nm})$ & No. of atoms & CPU times $(\mathrm{s})$ \\
\hline $10 \times 10$ & 3726 & 10.99 \\
$25 \times 25$ & 23548 & 67.68 \\
$45 \times 45$ & 76650 & 860.4 \\
$70 \times 70$ & 186632 & 4601.99 \\
\hline \hline
\end{tabular}

However, our predicted Poisson's ratio is larger than the value of 0.149 (Ref. 36) ( $a b$ initio models), the value of 0.227 (Ref. 37) (empirical force-constant calculations), and 0.186 (Ref. 17) (DFT). Furthermore, our predicted Poisson's ratio tends to be constant as the strain increases up to $10 \%$. After $15 \%$ strain, the Poissons ratio begins to increase slightly (from 0.41 to 0.46 , approximately). This behaviour is in contrast with the decreasing trends reported by Liu et al. ${ }^{17}$ and Kalosakas et al. ${ }^{38}$ These discrepancies may be attributed to the specific boundary conditions adopted in each case. We note that when constraining the horizontal displacements on the top and bottom edges of the SLGS (but keeping the left and right edges free to contract due to Poisson effects), our model predicts a slightly decreasing Poisson's ratio as the strain increases (simulation not shown here), with the same decreasing rate reported by Zhou et al. ${ }^{39}$ Furthermore, the boundary conditions applied to the sheet's edges may affect further the lateral contraction depending on the size of the SLGS. For instance, Liu et al. ${ }^{17}$ adopted a four-atom unit cell to perform their simulations. This small size might imply a very different behaviour when compared to the analysis of larger specimens (which is our case). Unfortunately, Liu et al. gave little details on how they apply the boundary conditions (they only specify that the lateral edges are free to move to allow Poisson contraction but did not give details on the specific boundary conditions applied to the other two edges). In addition, the theoretical treatment adopted for the analyses may also affect the behaviour of the Poisson's ratio. Liu et $a l .{ }^{17}$ performed DFT simulations, and our analyses considered the Tersoff-Brenner interatomic potential. It is important to note here that most of the values of Poisson's ratio calculated by $\mathrm{MM} / \mathrm{MD}$ tend to be significantly higher than those determined by DFT. $^{40}$

The advantages in computational efficiency of FE-based approaches with respect to atomistic simulations (e.g., MM, MD, and conjugate gradient) have been previously reported. ${ }^{41-43}$ We note that the computing times reported by Liu et $a l^{41}$ are almost identical to those for our FE simulations for the same number of atoms/nodes and similar computer hardware. Table III summarises the CPU times involved in the computational simulations of four SLGSs. The dimensions chosen for this study are $10 \mathrm{~nm} \times 10 \mathrm{~nm}, 25 \mathrm{~nm} \times 25 \mathrm{~nm}, 45 \mathrm{~nm}$ $\times 45 \mathrm{~nm}$, and $70 \mathrm{~nm} \times 70 \mathrm{~nm}$. For all these cases, a uniform tensile strain of $5 \%$ is applied with the boundary conditions shown in Figure 1. The computing times obtained in our simulations are in contrast with other methods, such as the standard conjugate gradient method, whose CPU times are almost two order of magnitude larger (refer to Ref. 41 for further details).

In conclusion, the present study demonstrates the potential capabilities of using an atomistic FE approach in conjunction with a hyperelasticity-based framework to investigate the mechanical behaviour of SLGSs. The simulations have been validated over a wide range of deformations, up to the onset of the breakage of the C-C bonds. It appears that the present approach could be exploited further in order to explore the mechanical behaviour of carbon nanostructures for a range of loading and boundary conditions.

${ }^{1}$ C. Li and T. Chou, Int. J. Solids Struct. 40, 2487 (2003).

${ }^{2}$ R. Ogden, Non-linear Elastic Deformations (Ellis Horwood, Chichester, 1984).

${ }^{3}$ E. Cadelano, P. L. Palla, S. Giordano, and L. Colombo, Phys. Rev. B 82, 235414 (2010).

${ }^{4}$ C. Lee, X. Wei, J. W. Kysar, and J. Hone, Science 321, 385 (2008).

${ }^{5}$ D. Brenner, Phys. Rev. B 42, 9458 (1990).

${ }^{6}$ E. I. Saavedra Flores, S. Adhikari, M. I. Friswell, and F. Scarpa, Comput. Mater. Sci. 50, 1083 (2011).

${ }^{7}$ K. Tserpes and P. Papanikos, Composites Part B 36, 468 (2005).

${ }^{8}$ ABAQUS, Analysis User's Manual. Version 6.10 (Dassault simulia, 2010).

${ }^{9}$ J. Marsden and T. Hughes, Mathematical Foundations of Elasticity (Prentice-Hall, New Jersey, 1983).

${ }^{10}$ A. Selvadurai, J. Mech. Phys. Solids 54, 1093 (2006).

${ }^{11}$ R. Hauptmann, K. Schweizerhof, and S. Doll, Int. J. Numer. Methods Eng. 49, 1121 (2000).

${ }^{12}$ S. Georgantzinos, D. Katsareas, and N. Anifantis, Int. J. Mech. Sci. 55, 85 (2012).

${ }^{13}$ S. Georgantzinos, G. Giannopoulos, D. Katsareas, P. Kakavas, and N. Anifantis, Comput. Mater. Sci. 50, 2057 (2011).

${ }^{14}$ A. Sakhaee-Pour, Solid State Commun. 149, 91 (2009).

${ }^{15}$ Z. Xu, J. Comput. Theor. Nanosci. 6, 625 (2009).

${ }^{16}$ R. Rasuli, A. Iraji Zad, and M. M. Ahadian, Nanotechnology 21, 185503 (2010).

${ }^{17}$ F. Liu, P. Ming, and J. Li, Phys. Rev. B 76, 064120 (2007).

${ }^{18}$ O. Frank, G. Tsoukleri, I. Riaz, K. Papagelis, J. Parthenios, A. C. Ferrari, A. K. Geim, K. S. Novoselov, and C. Galiotis, Nat. Commun. 2, 255 (2011).

${ }^{19}$ O. L. Blakslee, D. G. Proctor, E. J. Seldin, G. B. Spence, and T. Weng, J. Appl. Phys. 41, 3373 (1970).

${ }^{20}$ I. Frank, D. Tanenbaum, A. Van Der Zande, and P. McEuen, J. Vac. Sci. Technol. 25, 2558 (2007).

${ }^{21}$ M. Yu, B. Files, S. Arepalli, and R. Ruoff, Phys. Rev. Lett. 84, 5552 (2000).

${ }^{22}$ W. Ding, L. Calabri, K. M. Kohlhaas, X. Chen, D. A. Dikin, and R. S. Ruoff, Exp. Mech. 47, 25 (2007).

${ }^{23}$ H. Zhao and N. Aluru, Nano Lett. 9, 3012 (2009).

${ }^{24}$ Y. Jing, Y. Sun, H. Niu, and J. Shen, in Proceedings of the 13th International Conference on Fracture, Beijing, China, 2013.

${ }^{25}$ X. Ling and S. Atluri, J. Appl. Phys. 101, 064316 (2007).

${ }^{26}$ D. Robertson, D. Brenner, and J. Mintmire, Phys. Rev. B 45, 12592 (1992).

${ }^{27}$ T. Xiao and K. Liao, Phys. Rev. B 66, 153407 (2002).

${ }^{28}$ Q. Lu, W. Gao, and R. Huan, Modell. Simul. Mater. Sci. Eng. 19, 054006 (2011).

${ }^{29}$ J. Xiao, J. Staniszewski, and J. Gillespie, Jr., Compos. Struct. 88, 602 (2009).

${ }^{30} \mathrm{~J}$. Xiao and J. Gillespie, Jr., in Physics and Applications of Graphene Theory, edited by S. Mikhailov (InTech, 2011).

${ }^{31}$ A. Sears and R. Batra, Phys. Rev. B 69, 235406 (2004).

${ }^{32}$ M. Arroyo and T. Belytschko, Phys. Rev. B 69, 115415 (2004).

${ }^{33}$ C. Reddy, S. Rajendran, and K. Liew, Nanotechnology 17, 864 (2006).

${ }^{34}$ J. Zhou and R. Huang, J. Mech. Phys. Solids 56, 1609 (2008).

${ }^{35}$ Q. Lu and R. Huan, Int. J. Appl. Mech. 1, 443 (2009).

${ }^{36}$ K. N. Kudin and G. E. Scuseria, Phys. Rev. B 64, 235406 (2001).

${ }^{37}$ K. Michel and B. Verberck, Phys. Status Solidi B 245, 2177 (2008).

${ }^{38}$ G. Kalosakas, N. N. Lathiotakis, C. Galiotis, and K. Papagelis, J. Appl. Phys. 113, 134307 (2013).

${ }^{39}$ L. Zhou, Y. Wang, and G. Cao, J. Phys.: Condens. Matter 25, 125302 (2013).

${ }^{40} \mathrm{G}$. Cao, Polymers 6, 2404 (2014).

${ }^{41}$ B. Liu, H. Jiang, Y. Huang, H. Jiang, S. Qu, M. Yu, and K. Hwang, Phys. Rev. B 72, 035435 (2005).

${ }^{42}$ X. Xu and K. Liao, Mater. Phys. Mech. 4, 148 (2001).

${ }^{43}$ S. Rouhi and R. Ansari, Phys. E 44, 764 (2012). 